\title{
Microwave-Assisted Tandem Reactions towards a New Synthesis of Cyclohepta[b]chromene-9,11-diones
}

Joana L. C. Sousa

Artur M. S. Silva*

During the upload of the e-first version of this article, the letter 'v' was inadvertently added to the title. This has been corrected for the final online and print versions. 